\title{
The physiological changes of cumulative hemorrhagic shock in conscious rats
}

\author{
Tai Chu Peng ${ }^{1,2}$, Kuang Wen $\mathrm{Liao}^{3}$, Hui Ling Lai ${ }^{4}$, Yann Fen C. Chao ${ }^{5}$, \\ Fwu Mei Chang ${ }^{6}$, Horng-Jyh Harn ${ }^{1,7}$ \& Ru Ping Lee ${ }^{4,6,7, *}$ \\ ${ }^{1}$ Institute of Medical Sciences (TCP, HJH), Tzu Chi University, Hualien, Taiwan, R.O.C; ${ }^{2}$ Department of \\ Nursing (TCP), Tzu Chi College of Technology, Hualien, Taiwan, R.O.C; ${ }^{3}$ Department of Biological Science \\ and Technology (KWL), National Chiao Tung University, Hsinchu, Taiwan, R.O.C; ${ }^{4}$ Department of Nursing \\ (HLL, RPL), Tzu Chi General Hospital, Hualien, Taiwan, R.O.C; ${ }^{5}$ School of Nursing, National Yang Ming \\ University (YFCC), Taipei, Taiwan, R.O.C; ${ }^{6}$ Department of Nursing (FMC, RPL), Tzu Chi University, \\ Hualien, Taiwan, R.O.C; ${ }^{7}$ Neuro-Medical Scientific Center (HJH, RPL), Tzu Chi Medical Center, Hualien, \\ Taiwan, R.O.C
}

Received 7 September 2005; accepted 21 December 2005

(C) 2006 National Science Council, Taipei

Key words: animal model, conscious rats, cumulative hemorrhage, lactic acid, organ injury, unrestrained

\section{Summary}

Hemorrhagic shock is a common cause of death in emergency rooms. Current animal models of hemorrhage encounter a major problem that the volume and the rate of blood loss cannot be controlled. In addition, the use of anesthesia obscures physiological responses. Our experiments were designed to establish an animal model based on the clinical situation for studying hemorrhagic shock. Hemorrhagic shock was induced by withdrawing blood from a femoral arterial catheter. The blood volume withdrawn was $40 \%$ of the total blood volume for group 1 and $30 \%$ for group 2 and 3. Group 3 was anesthetized with sodium pentobarbital $(25 \mathrm{mg} / \mathrm{kg}$, i.v. $)$ at the beginning of blood withdrawal. Our data showed that the survival rate was $87.5 \%$ at $48 \mathrm{~h}$ in the conscious group and $0 \%$ at $9 \mathrm{~h}$ in anesthetic group after hemorrhage. The levels of mean arterial pressure, heart rate, white blood count, TNF- $\alpha$, IL1- $\beta$, CPK, and LDH after blood withdrawal in the anesthetic group were generally lower than those in conscious groups. These results indicated that anesthetics significantly affected the physiology of experimental animals. The conscious, unrestrained and cumulative volume-controlled hemorrhagic shock model was a good experimental model to investigate the physical phenomenon without anesthetic interfernce.

\section{Introduction}

Half of the patients with hemorrhagic shock die of substantial blood loss within $24 \mathrm{~h}$. Those who survive are at the high risk of developing infection and organ failure [1]. A number of animal models have been developed for simulating the end points of hemorrhagic shock. Methods used to control the hemorrhagic events include volume-controlled,

*To whom correspondence should be addressed. Phone $+886-$ 3-8560008; Fax: + 886-3-8574767; E-mail: fish@mail.tcu.edu.tw pressure-controlled, and uncontrolled hemorrhage models [2]. Fixed pressure [3] and fixed volume models $[4,5]$ of hemorrhage were developed and refined over the last century. These models were used in the development of standard therapeutic approaches. The uncontrolled hemorrhagic model has emerged recently and has been used increasingly for the last decade. However, the techniques used to initiate hemorrhage, such as catheters, tail amputation, aortotomy, and liver laceration, greatly influence the rate and the amount of blood loss [6]. Animal models which experience 
hemorrhage and tissue trauma require anesthetics to eliminate their pain. However, the effects of anesthetics can interfere with cardiovascular responses [7]. Some studies have shown that anesthetics suppress the protective sympathoexcitatory response. For example, opiates modulate hemodynamic instability, neuroendocrine secretion and cytokine responses to hemorrhagic shock [7, 8]. In addition, there is no consensus as to what volume loss and rate is most suitable in hemorrhagic shock [5]. An ideal experimental model of hemorrhagic shock should precipitate multiple organ dysfunctions and lead to multiple organ failure and late mortality [9]. How to measure these animals' arterial pressure (AP) and heart rate (HR) in these studies is essential. Accordingly, it is important in preclinical models to be able to develop an uncontrolled hemorrhagic model. Therefore, we designed a technique for continuous measurement of mean arterial pressure (MAP) and HR in conscious unrestrained rats, which can still freely move their bodies under such circumstances [10]. Our hypothesis was that a conscious unrestrained rat could decrease the interference of physiological changes from anesthetics and restraint. Moreover, blood withdrawal was easy to manipulate without inducing pain and fear to the animals. It also allowed us a longer period of time to observe the status of hemorrhagic shock.

\section{Materials and methods}

\section{Animal preparation}

Twenty-four male Wistar-Kyoto rats weighing 300-380 g were used in the study. The rats were purchased from the National Animal Center in Taiwan and housed in the animal center of Tzu Chi University. The room temperature was kept at $22 \pm 1^{\circ} \mathrm{C}$ and a $12 \mathrm{~h}$ light/dark cycle was maintained. Food and water were provided adequately. The experimental protocol was approved by the Animal Usage Regulation Committee of Tzu Chi University.

On the day before the experiment, animals were anesthetized by ether inhalation for about $15 \mathrm{~min}$. During the period of anesthesia, catheters (PE-50) were inserted in the left and right femoral arteries by aseptic surgical techniques. One catheter was connected to a pressure transducer (Gould
Instruments, USA) for recording AP and HR on a polygraph recorder (Power Lab, AD Instruments, USA); the other was connected to an infusion pump for blood withdrawal. The catheterization was completed within $15 \mathrm{~min}$, and the insertion wound was less than $0.5 \mathrm{~cm}^{2}$. After the insertion, each animal was placed on an individual metabolic plate overnight. Awakening soon after the operation, these rats were able to move freely and had access to water and food. They were kept on the plate during the experiment and their physiological changes were continuously monitored for $48 \mathrm{~h}$.

\section{Experimental protocol}

The animals were randomly divided into three groups. Arterial blood samples were obtained for baseline values before heparinization. Heparin (2 IU/gm BW) in $1 \mathrm{ml}$ normal saline was injected via the catheter into rats in $20 \mathrm{~min}$. Then blood withdrawal was conducted according to the withdrawal protocol. Hemorrhage was performed by drawing blood into a $10 \mathrm{ml}$ syringe from the femoral arterial catheter. An infusion pump to mimic the bleeding event controlled the withdrawal rate. The blood volume withdrawn was $40 \%$ of the total blood volume $(6 \mathrm{ml} / 100 \mathrm{gm} \mathrm{BW})$ for group 1 and $30 \%$ of the total blood volume for group 2 and 3, respectively [11-13]. The duration of blood withdrawal was $30 \mathrm{~min}$. Animals in group 1 and group 2 were kept in a conscious state without anesthesia. Group 3 was anesthetized with sodium pentobarbital $(25 \mathrm{mg} / \mathrm{kg}$, i.v.) before blood withdrawal and anesthesia was sustained by intravenous infusion of pentobarbital $(20 \mathrm{mg} /$ $\mathrm{kg} \mathrm{h}$ ). During the experiment, blood withdrawal in the same group was performed at the same time, and MAP and HR were continuously monitored. Additional blood samples for further analyses were taken from the femoral arterial catheter 1, 3, $6,9,12,18,24$, and $48 \mathrm{~h}$ after hemorrhage, and equal volumes of normal saline were replaced each time.

\section{Blood sample analyses}

The number of white blood cells (WBC) in the blood was measured immediately with the Sysmex K-1000 (NY, USA). Samples were then centrifuged at $12,000 \mathrm{rpm}$ for $5 \mathrm{~min}$. The supernatants 
were used to measure the concentrations of lactic acid, lactate dehydrogenase (LDH) and creatine phosphokinase (CPK) with an autoanalyzer (Vitros 750, Johnson \& Johnson, USA). Lactic acid was measured for evaluating the hemorrhagic shock, while CPK and LDH were for the organ injury.

\section{$T N F-\alpha$ and IL-1 $\beta$ measurement}

The concentrations of TNF- $\alpha$ and IL- $1 \beta$ in the samples were measured separately by an enzymelinked immunosorbent assay kit (ELISA, Endogen, USA). Blood samples were collected in plasma-separated tubes. All samples were stored at $-20^{\circ} \mathrm{C}$ before the tests. Reagents, samples and working standards were prepared according to the manufacturer's directions at the room temperature. The ELISA was performed following the manufacturer's instructions. Each sample was run in duplicate and determined by an automated ELISA reader at $450 / 540 \mathrm{~nm}$ wavelength.

\section{Data analysis}

All data were expressed as mean \pm SE. Nonparametric tests were used to evaluate differences between groups and time. Wilcoxon Signed Ranks Test was used to compare treatment effects at different times and the Mann-Whitney $U$ was used to compare treatment effects among different groups. A $p$ value less than 0.05 was considered statistically significant.

\section{Results}

The total blood volume withdrawn

There was no difference among groups in the means of body weight and MAP levels before the induction of hypovolemic shock. Group 1 was bled $8.5 \mathrm{ml}(40 \%$ of the total blood volume), while group 2 was bled $6.3 \mathrm{ml}$ and group 3, $6.8 \mathrm{ml}(30 \%$ of the total blood volume) at the initial blood withdrawal. After initial withdrawal, $0.8 \mathrm{ml}$ of blood was drawn for examination at each time point. Thus the cumulative blood withdrawal reached $50 \%$ at $3 \mathrm{~h}$ in group 1 and $9 \mathrm{~h}$ in group 2 and group 3 . The percentage of the total blood withdrawn at $24 \mathrm{~h}$ was $68.7 \%$ in group 1 and $61.2 \%$ in group 2 (Table 1). After hemorrhage, the survival rates of group 1 were $100 \%$ at $24 \mathrm{~h}$ and $87.5 \%$ at $48 \mathrm{~h}$, which were the same as those of group 2. The survival rate in the anesthetic group was $75 \%$ at $6 \mathrm{~h}, 50 \%$ at $8 \mathrm{~h}$ and $0 \%$ after $9 \mathrm{~h}$.

\section{The mean arterial pressure and $H R$}

The averages of MAP during the basal period did not show any significant difference among three groups $(124 \pm 1 \mathrm{mmHg}$ for group $1,129 \pm 4 \mathrm{mmHg}$ for group 2 and $127 \pm 4 \mathrm{mmHg}$ for group 3 before anesthesia) (Figure 1a). After the initial blood withdrawal, MAP quickly decreased to $36 \pm 3$ $\mathrm{mmHg}(30 \%$ of baseline level) in group 1, $53 \pm 9 \mathrm{mmHg}$ (49\% of baseline level) in group 2

Table 1. The volume of cumulative blood withdrawal in $24 \mathrm{~h}$.

\begin{tabular}{|c|c|c|c|c|c|c|c|c|c|}
\hline Item & Baseline & Initial draw & $1 \mathrm{~h}$ & $3 \mathrm{~h}$ & $6 \mathrm{~h}$ & $9 \mathrm{~h}$ & $12 \mathrm{~h}$ & $18 \mathrm{~h}$ & $24 \mathrm{~h}$ \\
\hline \multicolumn{10}{|l|}{ Group 1 (40\% blood loss) } \\
\hline Blood withdrawal (ml) & 0.8 & 8.5 & 0.8 & 0.8 & 0.8 & 0.8 & 0.8 & 0.8 & 0.8 \\
\hline Total blood loss (ml) & 0.8 & 9.3 & 10.1 & 10.9 & 11.7 & 12.5 & 13.1 & 13.9 & 14.7 \\
\hline ( $\%$ of whole blood volume) & (3.8) & $(43.8)$ & $(47.6)$ & $(50.9)$ & $(54.7)$ & $(58.8)$ & $(61.2)$ & $(64.9)$ & $(68.7)$ \\
\hline \multicolumn{10}{|l|}{ Group 2 (30\% blood loss) } \\
\hline Blood withdrawal (ml) & 0.8 & 6.3 & 0.8 & 0.8 & 0.8 & 0.8 & 0.8 & 0.8 & 0.8 \\
\hline Total blood loss (ml) & 0.8 & 7.1 & 7.9 & 8.7 & 9.5 & 10.3 & 11.1 & 11.9 & 12.7 \\
\hline ( $\%$ of whole blood volume) & $(4.2)$ & $(34.2)$ & $(38.1)$ & (41.9) & $(45.8)$ & (49.6) & $(53.5)$ & $(57.3)$ & $(61.2)$ \\
\hline \multicolumn{10}{|c|}{ Group 3 (Anesthesia, 30\% blood loss) } \\
\hline Blood withdrawal (ml) & 0.8 & 6.8 & 0.8 & 0.8 & 0.8 & 0.8 & & & \\
\hline Total blood loss (ml) & 0.8 & 7.6 & 8.4 & 9.2 & 10.0 & 10.8 & & & \\
\hline ( $\%$ of whole blood volume) & $(3.5)$ & $(33.3)$ & $(36.8)$ & $(40.4)$ & (43.9) & $(47.4)$ & & & \\
\hline
\end{tabular}


and $52 \pm 6 \mathrm{mmHg}(41 \%$ of baseline $)$ in group 3 within $30 \mathrm{~min}$. However, the MAP values in group 1 and 2 gradually rose, though still lower than the baseline, whereas the average values of MAP in the anesthetic group rose slightly at $1 \mathrm{~h}$ $(67 \pm 7 \mathrm{mmHg})$, but it gradually decreased to $30 \%$ of the baseline at $6 \mathrm{~h}(39 \pm 4 \mathrm{mmHg})$. Consequently, the animals in the anesthetic group died $9 \mathrm{~h}$ after hemorrhage (Figure 1a). The baseline values of HR in group 1 (398 $\pm 25 \mathrm{bpm})$ and group
$2(417 \pm 41 \mathrm{bpm})$ were not significantly different (Figure 1b). HR values increased quickly in both group 1 and group 2 after the initial blood withdrawal $(457 \pm 36 \mathrm{bpm}$ for group 1 and $477 \pm 44 \mathrm{bpm}$ for group 2 at $1 \mathrm{~h}$ ) and remained significantly higher than the baseline value at 12 , 18,24 , and $48 \mathrm{~h}(p<0.05)$. However, the HR values in the anesthetic group decreased gradually from $380 \pm 44 \mathrm{bpm}$ to $274 \pm 13 \mathrm{bpm}$ at $1 \mathrm{~h}$ after blood withdrawal with anesthesia.
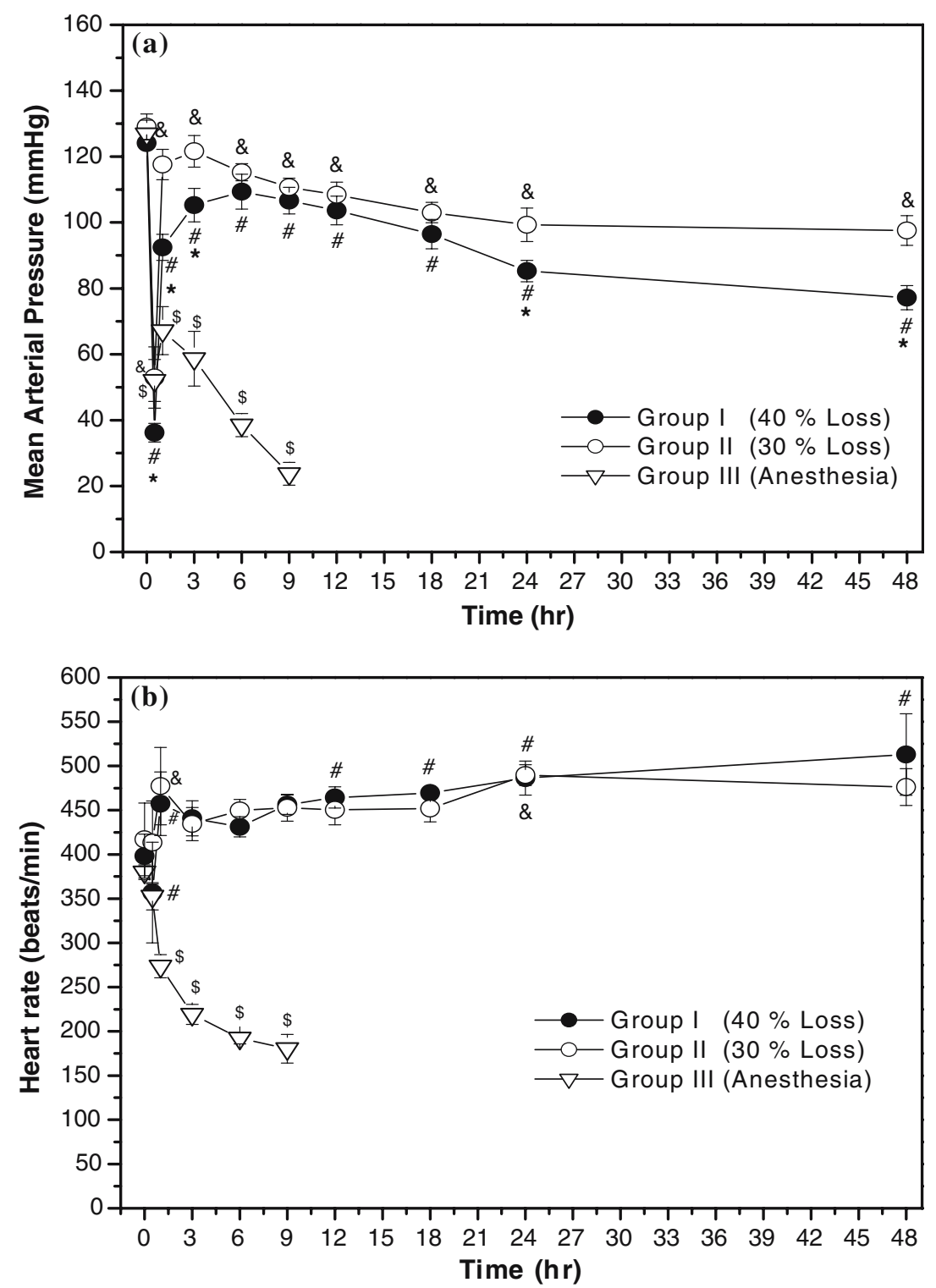

Figure 1. The values of MAP (a) and HR (b) at different time points. ${ }^{*} p<0.05$ indicates the $40 \%$ blood loss group (group 1 ) compared with the $30 \%$ blood loss group (group 2). ${ }^{*} p<0.05$ indicates MAP and HR of group 1 at different time points compared with its basal level $(0 \mathrm{~h}) .{ }^{\&} p<0.05$ indicates MAP and HR of group 2 at different time points compared with its basal level. ${ }_{p}<<0.05$ indicates MAP and HR of group 3 at different time points compared with its basal level. 


\section{Plasma lactic acid levels}

The baseline levels of lactic acid in plasma were not significantly different among the groups $(0.94 \pm 0.03 \mathrm{mmol} / 1$ in group $1,0.94 \pm 0.07 \mathrm{mmol} / 1$ in group 2 and $1.10 \pm 0.04 \mathrm{mmol} / \mathrm{l}$ in the anesthetic group) (Figure 2). Hemorrhage in group 1 resulted in a twofold increase of lactic acid (1.94 \pm $0.36 \mathrm{mmol} / \mathrm{l})$ at $9 \mathrm{~h}$ and a threefold increase $(2.83 \pm 0.45 \mathrm{mmol} / \mathrm{l})$ at $18 \mathrm{~h}$ compared with the baseline level $(p<0.05)$. Although the values decreased after $24 \mathrm{~h}$, they remained at a higher level until $48 \mathrm{~h}$. In contrast, the lactic acid levels in group 2 significantly increased after $24 \mathrm{~h}$ $(p<0.05)$. In the anesthetic group, however, the values of lactic acid remained lower than the basal level after blood withdrawal but dramatically increased before death (from $1.1 \pm 0.04$ to $4.9 \pm$ $0.56 \mathrm{mmol} / \mathrm{l})$.

\section{White blood cell and plasma cytokine levels}

Total leukocyte count increased immediately in all groups at $1 \mathrm{~h}$. It decreased at 3, 6, 9, and $12 \mathrm{~h}$ in group 1 and the lowest level occurred at $6 \mathrm{~h}$ (Figure 3a). The amount recovered progres- sively to the basal level from 9 to $18 \mathrm{~h}$. In group 2, leukocytes decreased at 3, 6, 9, 12, 18, and $24 \mathrm{~h}$ after initial blood withdrawal and had become lower than those of group 1 since $9 \mathrm{~h}$. The lowest level occurred at $9 \mathrm{~h}$ but it recovered to the basal level from 12 to $24 \mathrm{~h}$. The leukocyte count of the anesthetic group had decreased since $3 \mathrm{~h}$ and had never recovered ever since.

Mean cytokine levels were similar between groups before hemorrhage. Plasma TNF- $\alpha$ level gradually elevated to $47.5 \%$ above the basal level at $9 \mathrm{~h}$ after the completion of initial hemorrhage in group 1, and then decreased to the basal level (Figure 3b). The TNF- $\alpha$ level elevated 1.8-fold at $18 \mathrm{~h}$ after initial hemorrhage in group 2. However, the anesthetic group showed no significant change after initial hemorrhage. The plasma IL-1 $\beta$ level significantly elevated after initial hemorrhage in group 1 (Figure 3c). When compared with the basal level, it increased by $73 \%$ at $9 \mathrm{~h}$ but decreased to the baseline at $12 \mathrm{~h}$. In group 2, IL$1 \beta$ level was elevated by $59 \%$ at $12 \mathrm{~h}$ after initial hemorrhage. The IL-1 $\beta$ level of the anesthetic group showed no significant change after initial hemorrhage.

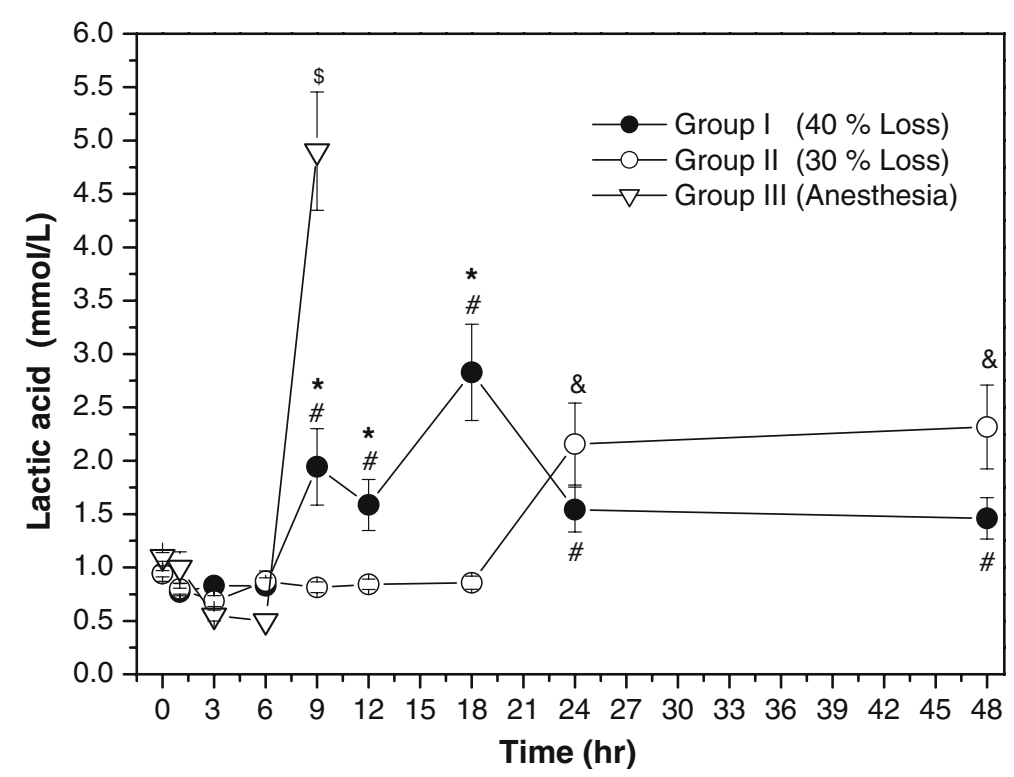

Figure 2. The change of lactic acid between $40 \%$ blood loss group (group 1), 30\% blood loss group (group 2) and the anesthetic group (group 3). ${ }^{*} p<0.05$ indicates group 1 compared with group 2. ${ }^{\#} p<0.05$ indicates the lactic acid of group 1 at different time points compared with its basal level $(0 \mathrm{~h}) .{ }^{\&} p<0.05$ indicates the lactic acid of group 2 at different time points compared with its basal level. ${ }^{\$} p<0.05$ indicates the lactic acid of group 3 at different time points compared with its basal level. 

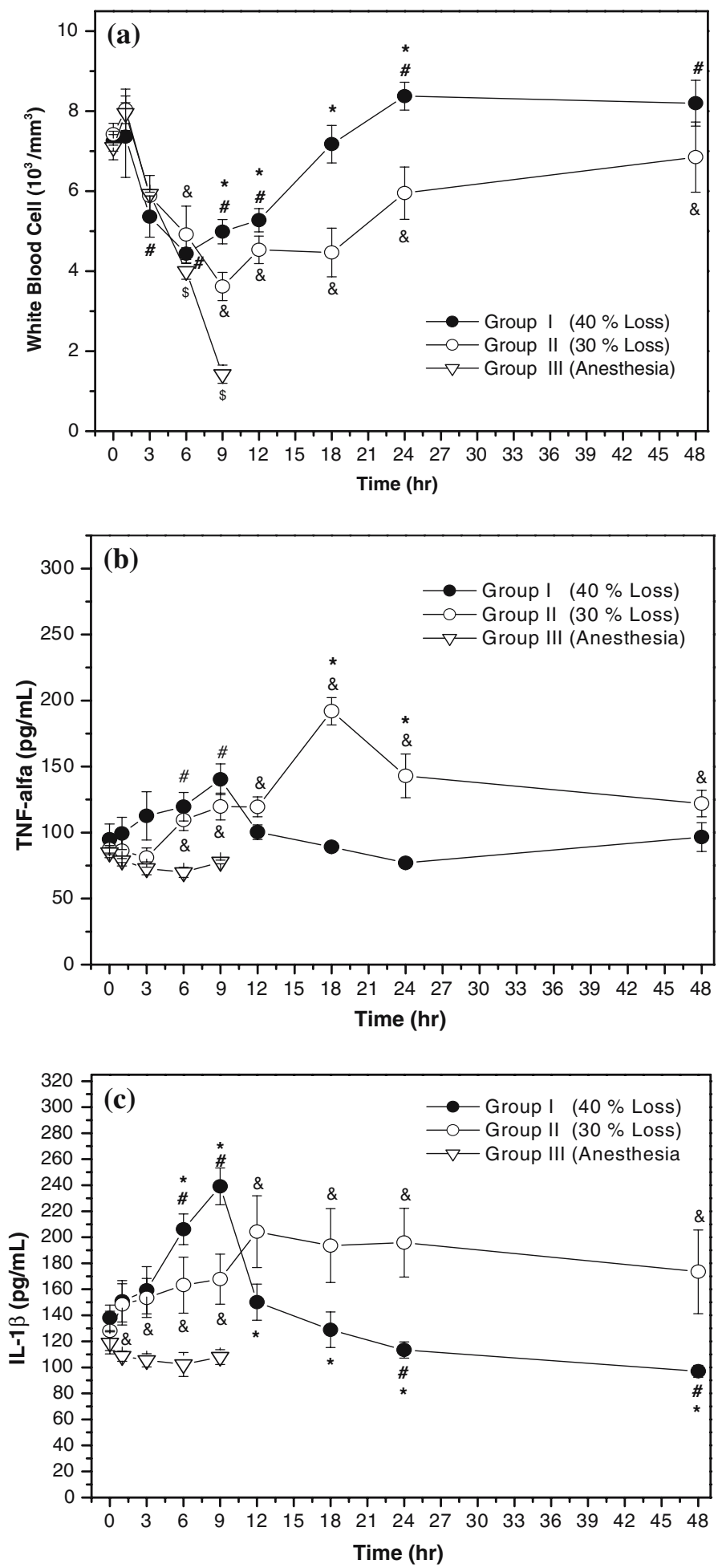

Figure 3. Changes of WBC (a), TNF- $\alpha$ (b) and IL1- $\beta$ (c) levels. ${ }^{*} p<0.05$ indicates the $40 \%$ blood loss group (group 1 ) compared with the $30 \%$ group (group 2). ${ }^{\#} p<0.05$ indicates changes of group 1 at different time points compared with its basal level $(0 \mathrm{~h})$. ${ }^{\&} p<0.05$ indicates changes of group 2 at different time points compared with its basal level. ${ }^{{ }} p<0.05$ indicates changes of group 3 at different time points compared with its basal level. 
Effects of hemorrhage on CPK and LDH changes

Values of blood CPK in group 1 had increased since $3 \mathrm{~h}$ after initial blood withdrawal and tripled at $9 \mathrm{~h}$, higher than the values in group 2 during the observation period (Figure 4a). Values of blood LDH had also increased since $3 \mathrm{~h}$ after initial blood withdrawal in group 1 (Figure $4 b$ ). The LDH for group 1 peaked at $9 \mathrm{~h}$ and decreased to the baseline at $48 \mathrm{~h}$. However, it remained higher than that in group 2 at 3, 6, 9, 12, 18, and $24 \mathrm{~h}$. In the anesthetic group, the values of CPK and LDH were lower than their baseline level after blood withdrawal.

\section{Discussion}

Most animal models for uncontrolled hemorrhagic shock achieve blood withdrawal by tail amputation
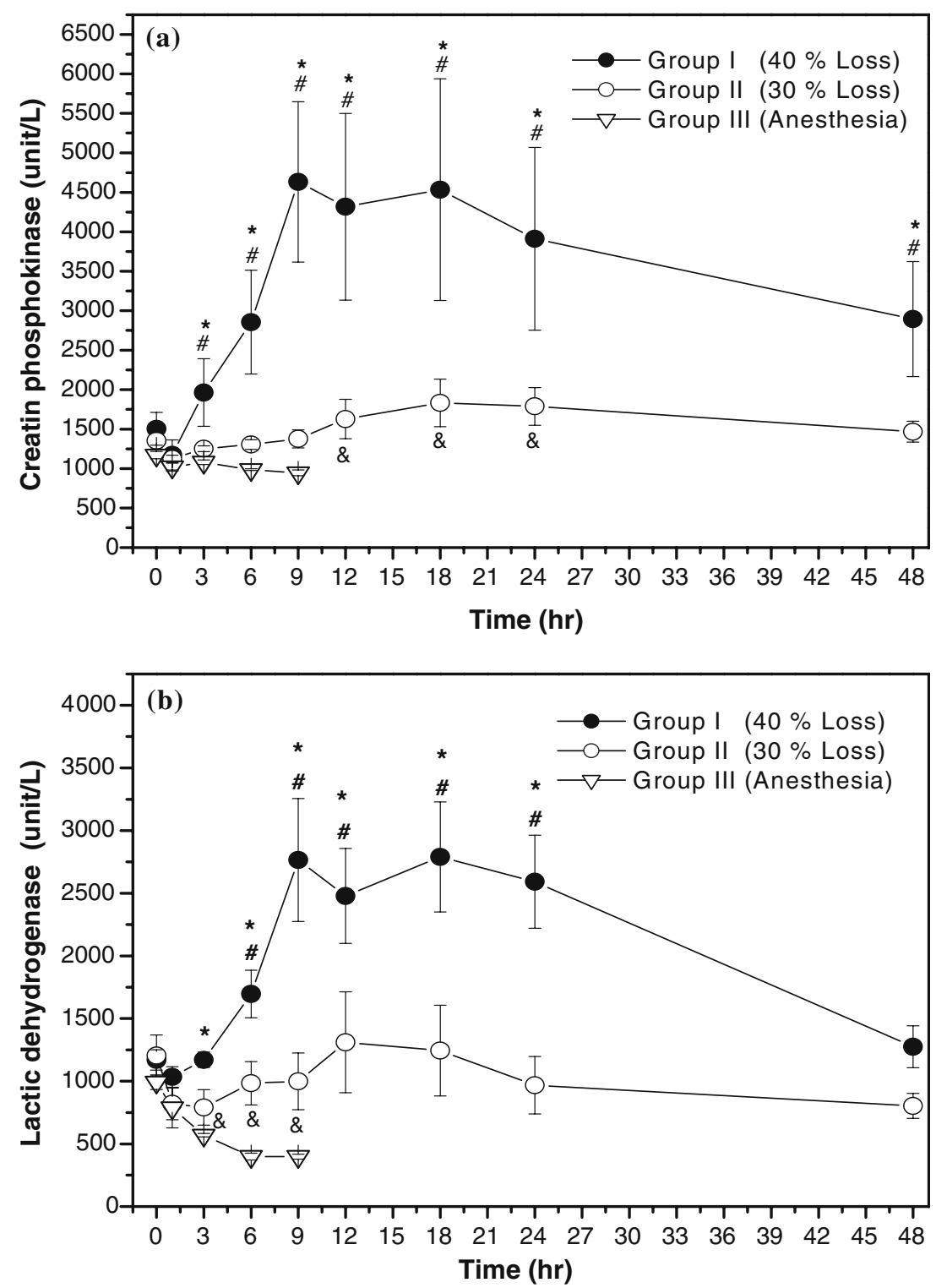

Figure 4. Changes of creatine phosphokinase (CPK) and lactic dehydrogenase (LDH) levels. ${ }^{*} p<0.05$ indicates group 1 compared with group 2. ${ }^{\#} p<0.05$ indicates changes of group 1 at different time points compared with its basal level $(0 \mathrm{~h}) .{ }^{\&} p<0.05$ indicates changes of group 2 at different time points compared with its basal level. 
[14], or liver or spleen laceration [15, 16]. These procedures greatly influence the rate and the amount of blood loss and require a long period of anesthetic or fasting state. In our study a catheter was implanted in each femoral artery of the rat, which allowed blood withdrawal and monitoring of the MAP and HR to be performed simultaneously. In this model, the animal was kept unrestrained and could freely move its body. Besides, blood withdrawal of eight rats was performed at the same time to avoid the variation of data from the procedure [10, 17]. In addition, blood withdrawal from the femoral artery catheter could be performed for as long as $48 \mathrm{~h}$ while blood loss measurement was being taken. Moreover, the animals perceived no pain and did not struggle when their blood was withdrawn and the animals were kept conscious without anesthesia and restraint. We believe the animals experienced less stress during the experiment, thereby minimizing the contribution made by stress that may be present at the pathophysiological changes seen in hemorrhagic shock $[6,7]$.

Many researchers agree that $50 \%$ blood loss is a critical point in hemorrhagic shock [6]. In 1991, Singh used pressure-fixed control model in their study in which the initial total blood volume removed was about $60.9 \pm 0.2 \%$ [18]. In our model, the cumulative blood withdrawal volume reached $50 \%$ at $3 \mathrm{~h}$ in group 1 and $9 \mathrm{~h}$ in group 2, and reached $61.2 \%$ at $12 \mathrm{~h}$ in group 1 and $24 \mathrm{~h}$ in group 2. Hemorrhage caused a rapid fall in the MAP, which reached an average of $36 \pm 3 \mathrm{mmHg}$ in group 1, equivalent to that seen in the fixed pressure model $[8,19]$. Subsequently, the MAP gradually returned to the baseline and decreased at $6 \mathrm{~h}$ in group 1. The MAP of group 1 was significantly lower than that of group 2 (63 \pm $9 \mathrm{mmHg}$ ) and it did not return at all in the anesthetic group.

Shock is now being defined at the cellular level as the inadequate delivery of oxygen to the cells of the body [20]. A recent study demonstrated that cumulative oxygen debt, arterial base excess and plasma lactate are superior predictors of outcome in comparison to such variables as the volume of blood loss, blood pressure and cardiac output [1, 21]. The onset of hemorrhage is associated with a shift to metabolic acidosis. This metabolic acidosis is the most likely result of tissue hypoperfusion and anaerobic metabolism, leading to a significant increase in circulating lactate levels. Blood lactate levels in hemorrhagic shock are used increasingly as reliable indicators of the severity of injury and the adequacy of fluid resuscitation [21, 22]. In our model, cumulative hemorrhage resulted in a twofold plasma lactic acid increase at $9 \mathrm{~h}$ and threefold at $18 \mathrm{~h}$ in group 1, which was higher and increased earlier than those in group 2. This could be caused by decompensation. Therefore, the model of $40 \%$ blood withdrawal (group 1) could be applied to a sub-acute hemorrhagic shock study focusing on the first $6 \mathrm{~h}$.

Acute hemorrhage leads to the activation of humoral systems, such as complement, coagulation, fibrinolysis, and the kallikrein-kinin system, resulting in the release and synthesis of various activation products. On the early stage, platelets and polymorphonuclear leukocytes (PMN) are chiefly affected. Released mediators of activated cells cause a systemic inflammatory reaction and thus lead to organ damage [23, 24]. TNF- $\alpha$ and IL1- $\beta$ play a major role in systemic inflammatory response. They also enhance leukocyte-endothelial cell adhesion, stimulate chemokine secretion, and induce neutrophil activation [25]. In addition, TNF- $\alpha$ is increased after hemorrhagic shock [26]. In this study, TNF- $\alpha$ and IL1- $\beta$ were increased before $12 \mathrm{~h}$ and peaked at $9 \mathrm{~h}$, while total leukocyte count was lowest at $6 \mathrm{~h}$ after hemorrhage in group 1 . These data indicated that tissue ischemia after hemorrhage might directly stimulate TNF- $\alpha$ and IL1- $\beta$ release. The TNF- $\alpha$ and IL1- $\beta$ might act as mediators in the pathophysiological changes of hemorrhagic shock.

Exposure of laboratory animals to anesthetic agents might change the immunity function, including the production of cytokines and the reduction in natural killer cell activity [27-29]. The hemodynamics was also changed after anesthesia $[30,31]$. In the present study, the decrease in WBC in 1-6 h after blood withdrawal occurred in conscious and anesthetize rats but the increase of TNF- $\alpha$ and IL-1 $\beta$ after blood withdrawal was observed only in conscious rats but not in anesthetized rats which suggest a stronger inflammatory response after hemorrhage may occur in conscious animals. However, the values of MAP and HR in the anesthetic group were lower than those in the conscious group and the animals died before $9 \mathrm{~h}$. 
The pathophysiological change of hemorrhagic shock is closely related to the development of systemic inflammatory response syndrome (SIRS), which can lead to a subsequent organ dysfunction syndrome, and result in multiple organ dysfunction syndrome (MODS). After hemorrhage, the blood CPK and LDH concentrations were significantly increased in conscious animal groups but remained at a lower level in the anesthetic group. These results suggest that anesthesia retarded proinflammatory response in terms of a reduced defense reaction against hemorrhagic shock. LDH is commonly used as an indicator for injury of the liver, heart and kidney, while CPK is used for heart and skeletal muscle. Our hemorrhagic shock model was associated with multiple organ dysfunctions and therefore may be useful for the study of SIRS and MODS.

Hemorrhage causes activation of sympathetic outflow to elevate epinephrine and norepinephrine levels in circulation [32] and was associated with a rise in circulating beta-endorphin. In addition, an early increase in tissue cytokine content was initiated by hemorrhagic shock, which is associated with elevations in circulating epinephrine and norepinephrine [33]. Norepinephrine is a more potent inhibitor of TNF- $\alpha$. In contrast, TNF- $\alpha$ is increased after hemorrhagic shock [26]. These results demonstrate that the effects of norepinephrine are protective from tissue injury but are likely to contribute to the generalized immunosuppression following blood withdrawal [32]. In addition, hemorrhage enhances the expression of epinephrine and norepinephrine which can activate the sympathetic system that further increases the HR and AP, leading to more oxygen delivery and tissue perfusion, and less cellular hypoxia, organ damage, and death. Therefore, sympathetic activation provided certain benefits for the damage induced by blood withdrawal. However, many other factors were involved in animal mortality induced by hemorrhage. The inflammatory cytokine release (such as TNF- $\alpha$ and IL-1 $\beta$ ) has been reported to be associated with tissue damage while other factors with tissue or organ damage. Beside the inflammatory cytokine release, the loss of intravascular volume also lead sequentially to hemodynamic instability, decreases in oxygen delivery, decreased tissue perfusion, cellular hypoxia, organ damage, and death. In this study, although pentobarbital lowers inflammatory cytokine release to decrease tissue damage, it also suppresses sympathetic activity to cause cellular hypoxia. Therefore, this study can provide a good animal model to investigate the relation between anesthetics, cytokine release and sympathetic activity.

\section{Conclusions}

Animal models utilizing anesthesia have been used in a number of studies of hemorrhagic shock. The anesthetic drugs may interfere with the physiologic course of hemorrhagic shock and make this model unlike that seen clinically. On the other hand, in previous uncontrolled hemorrhagic shock animal models, the rate and the volume of blood loss could be adequately controlled and required prolonged anesthetic or a fasting state. In addition, these models cannot measure physiologic parameters continuously at given intervals for a prolonged period. Use of this conscious, unrestrained and less stressed rat model makes the control of blood loss more convenient and it could continuously monitor MAP and HR and collect blood samples for analysis for as long as $48 \mathrm{~h}$. Moreover, the $40 \%$ blood withdrawal could be chosen for study of a sub-acute hemorrhagic shock. The therapeutic window is suggested on the first $6 \mathrm{~h}$ after hemorrhage.

\section{Acknowledgements}

This work was supported in part by grants from the National Science Council (93-2314-B-320$010)$. The authors gratefully thank Mr. Shu Jang Kou for his excellent technical advice, and thank Mike Biological Technologies Ins. for their equipment assistance in this study.

\section{References}

1. Rixen D., Raum M., Holzgraefe B. et al., A pig hemorrhagic shock model: oxygen debt and metabolic acidemia as indicators of severity. Shock 16: 239-244, 2001.

2. Deitch E.A., Animal models of sepsis and shock: a review and lessons learned. Shock 9: 1-11, 1998.

3. Wiggers C.J., The present status of the shock problem. Physiol. Rev. 22: 74-123, 1942.

4. Chintala M.S. and Jandhyala B.S., Comparative evaluation of the effects of felodipine, hydralazine, and naloxone on 
the survival rate in rats subjected to a "fixed volume" model of hemorrhagic shock. Circ. Shock 32: 219-229, 1990.

5. Bitterman H., Reissman P., Bitterman N. et al., Oxygen therapy in hemorrhagic shock. Circ. Shock 33: 183-191, 1991.

6. Majde J.A., Animal models for hemorrhage and resuscitation research. J. Trauma 54: s100-s105, 2003.

7. Schadt J.C. and Ludbrook J., Hemodynamic and neurohumoral resposes to acute hypovolemia in conscious mammals. Am. J. Physiol. 260: H305-H318, 1991.

8. Molina P.E., Opiate modulation of hemodynamic, hormonal, and cytokine responses to hemorrhage. Shock 15: 471-478, 2001.

9. McCance K.L. and Huether S.E., Pathophysiology, 4th ed., Mosby, Inc., St. Louis, Missouri USA, 2002, pp. 450-470.

10. Lee R.P., Wang D., Lin N.T. et al., A modified technique for tail-cuff pressure measurement in unrestrained rats at conscious state. J. Biomed. Sci. 9: 424-427, 2002.

11. Van Zutphen L.M.F., Baumans V. and Beynen A.C., Principles of Laboratory Animal Science: a Contribution to the Humane Use and Care of Animals to the Quality of Experiment Results. Elservier, Amsterdam, 1993, pp. 1774.

12. Takasu A., Prueckner S., Tisherman S.A. et al., Effects of increased oxygen breathing in a volume controlled hemorrhagic shock outcome model in rats. Resuscitation 45: 209220, 2000

13. Capone A., Safar P., Stezoski S.W. et al., Uncontrolled hemorrhagic shock outcome model in rats. Resuscitation 29: 143-152, 1995.

14. Marshall H.P. Jr., Capone A., Courcoulas A.P. et al., Effects of hemodilution on long-term survival in an uncontrolled hemorrhagic shock model in rats. J. Trauma 43: 673-679, 1997.

15. Solomonov E., Hirsh M., Yahiya A. et al., The effect of vigorous fluid resuscitation in uncontrolled hemorrhagic shock after massive splenic injury. Crit. Care Med. 28: 749754, 2000

16. Raedler C., Voelckel W.G., Wenzel V. et al., Treatment of uncontrolled hemorrhagic shock after liver trauma: fatal effects of fluid resuscitation versus improved outcome after vasopressin. Anesth. Analg. 98: 1759-1766, 2000.

17. Lee R.P., Wang D., Lin N.T. et al., Physiological and chemical indicators for early and late stages of sepsis in conscious rats. J. Biomed. Sci. 9: 613-621, 2002.

18. Singh G., Chaudry K.I. and Chaudry I.H., Crystalloid is as effective as blood in the resuscitation of hemorrhagic shock. Ann. Surg. 215: 377-382, 1992.

19. Flohe S., Ackermann M., Reuter M. et al., Sublethal hemorrhagic shock reduces tumor necrosis factor-alpha- producing capacity in different cell compartments. Eur. Cytokine Netw. 11: 420-426, 2000.

20. Moore K.E. and Murtaugh R.J., Pathophysiologic characteristics of hypovolemic shock. Vet. Clin. North Am. Small Anim. Prac. 31: 1115-1128, 2001.

21. Dunham C.M., Siegel J.H., Weireter L. et al., Oxygen debt and metabolic acidemia as quantitative predictors of mortality and the severity of the ischemic insult in hemorrhagic shock. Crit. Care Med. 19: 231-243, 1991.

22. Claridge J.A., Enelow R.I. and Young J.S., Hemorrhage and resuscitation induce delayed inflammation and pulmonary dysfunction in mice. J. Surg. Res. 92: 206-213, 2000.

23. Bone R.C., Balk R.A., Cerra F.B. et al., Definitions for sepsis and organ failure and guidelines for the use of innovative therapies in sepsis. Chest 101: 1644-1655, 1992.

24. Marshall J.C., Inflammation, coagulopathy, and the pathogenesis of multiple prgan dysfunction syndrome. Crit. Care Med. 29: s99-s106, 2001.

25. Zingarelli B., Squadrito F., Altavilla D. et al., Role of tumor necrosis factor-alpha in acute hypovolemic hemorrhagic shock in rats. Am. J. Physiol. 266: H1512-1515, 1994.

26. Rhee P., Waxman K., Clark L. et al., Tumor necrosis factor and monocytes are released during hemorrhagic shock. Resuscitation 25: 249-255, 1993.

27. Kelbela I. and Weiss M., Anaesthetics and immune function. Anaesthesiology 14: 685-691, 2001.

28. Kotani N., Hashimoto H., Sessler D.I. et al., Intraoperative modulation of alveolar macrophage function during isoflurane and propofol anesthesia. Anesthesiology 89: 11251132,1998

29. Kotani N., Hashimoto H., Sessler D.I. et al., Expression of genes for proinflammatory cytokines in alveolar macrophages during propofol and isoflurane anesthesia. Anesth. Analg. 89: 1250-1256, 1999.

30. Xu H., Aibiki M., Yokono S. et al., Dose-depenndent effects of propofol on renal sympathetic nerve activity, blood pressure and heart rate in urethane-anesthetized rabbits. Eur. J. Pharmacol. 387: 79-85, 2000.

31. Loeb A.L., Godeny I. and Longnecker D.E., Anesthestics alter relative contributions of $\mathrm{NO}$ and $\mathrm{EDHF}$ in rat cremaster muscle microcirculation. Am. J. Physiol. Heart Circ. Physiol. 273: 618-627, 1997.

32. Molina P.E., Noradrenergic inhibition of TNF upregulation in hemorrhagic shock. Neuroimmunomodulation 9: 125-33, 2001.

33. Molina P.E., Malek S., Lang C.H., Qian L., Naukam R. and Abumrad N.N., Early organ-specific hemorrhageinduced increases in tissue cytokine content: associated neurohormonal and opioid alterations. Neuroimmunomodulation 4(1) 28-36, 1997, Jan-Feb. 